Check for updates

Cite this: Chem. Sci., 2021, 12, 14198

๑ All publication charges for this article have been paid for by the Royal Society of Chemistry

Received 19th May 2021

Accepted 5th October 2021

DOI: $10.1039 / \mathrm{d} 1 \mathrm{sc} 02729 \mathrm{~h}$

rsc.li/chemical-science

\title{
Large polarization and record-high performance of energy storage induced by a phase change in organic molecular crystals $\uparrow$
}

\author{
Sachio Horiuchi (D) *a and Shoji Ishibashi (D) ${ }^{b}$
}

Dielectrics that undergo electric-field-induced phase changes are promising for use as high-power electrical energy storage materials and transducers. We demonstrate the stepwise on/off switching of large polarization in a series of dielectrics by flipping their antipolar or canted electric dipoles via proton transfer and inducing simultaneous geometric changes in their $\pi$-conjugation system. Among antiferroelectric organic molecular crystals, the largest-magnitude polarization jump was obtained as 18 $\mu \mathrm{C} \mathrm{cm} \mathrm{cm}^{-2}$ through revisited measurements of squaric acid (SQA) crystals with improved dielectric strength. The second-best polarization jump of $15.1 \mu \mathrm{C} \mathrm{cm}^{-2}$ was achieved with a newly discovered antiferroelectric, furan-3,4-dicarboxylic acid. The field-induced dielectric phase changes show rich variations in their mechanisms. The quadruple polarization hysteresis loop observed for a 3-(4chlorophenyl)propiolic acid crystal was caused by a two-step phase transition with moderate polarization jumps. The ferroelectric 2-phenylmalondialdehyde single crystal having canted dipoles behaved as an amphoteric dielectric, exhibiting a single or double polarization hysteresis loop depending on the direction of the external field. The magnitude of a series of observed polarizations was consistently reproduced within the simplest sublattice model by the density functional theory calculations of dipole moments flipping over a hydrogen-bonded chain or sheet (sublattice) irrespective of compounds. This finding guarantees a tool that will deepen our understanding of the microscopic phase-change mechanisms and accelerate the materials design and exploration for improving energystorage performance. The excellent energy-storage performance of SQA was demonstrated by both a high recoverable energy-storage density $W_{r}$ of $3.3 \mathrm{~J} \mathrm{~cm}^{-3}$ and a nearly ideal efficiency (90\%). Because of the low crystal density, the corresponding energy density per mass $W_{r}^{\prime}\left(1.75 \mathrm{~J} \mathrm{~g}^{-1}\right)$ exceeded those derived from the highest $W_{\mathrm{r}}$ values $\left(\sim 8-11 \mathrm{~J} \mathrm{~cm}^{-3}\right)$ reported for several bulk antiferroelectric ceramics $\left(W_{r}^{\prime}<1.3 \mathrm{~J} \mathrm{~g}^{-1}\right)$, without modification to relaxor forms.

\section{Introduction}

Highly polarizable dielectrics have been used in diverse electronic, mechatronic, and optoelectronic applications., ${ }^{\mathbf{1} 2}$ In particular, phase-change dielectrics accompanied by a large polarization jump are desired for high-power electrical energy storage, which is increasingly demanded with the expansion of modern commercialization., ${ }^{3,4}$ One of the most suitable dielectrics is the antiferroelectric, ${ }^{5,6}$ in which permanent dipoles can

${ }^{a}$ Research Institute for Advanced Electronics and Photonics (RIAEP), National Institute of Advanced Industrial Science and Technology (AIST), Tsukuba, Ibaraki 305-8565, Japan

${ }^{b}$ Research Center for Computational Design of Advanced Functional Materials (CDFMat), National Institute of Advanced Industrial Science and Technology (AIST), Tsukuba 305-8568, Japan

$\dagger$ Electronic supplementary information (ESI) available: Additional structural information, CIF files, and additional data of theoretical calculations. CCDC 2082229-2082232. For ESI and crystallographic data in CIF or other electronic format see DOI: $10.1039 / \mathrm{d} 1 \mathrm{sc} 02729 \mathrm{~h}$ be reversibly switched between antiparallel and parallel arrangements by changing the amplitude of an externally applied electric field. ${ }^{7-11}$ The reversibility between the antiferroelectric (AFE) and ferroelectric (FE) phases yields electric polarization $(P) v s$. electric field $(E)$ hysteresis, where the $P-E$ curves exhibit double loops instead of the single loop exhibited by ferroelectrics. The stored energy density $W_{\mathrm{s}}$ during the forward (antiferroelectric-to-ferroelectric phase) switching, the recoverable energy density $W_{\mathrm{r}}$ during the backward (ferroelectric-to-antiferroelectric AFE phase) switching, and the efficiency $\eta$ can be evaluated through numerical integration of the $P-E$ curves according to the following equations:

$$
\begin{gathered}
W_{\mathrm{s}}=\int_{0}^{P_{\mathrm{m}}} E \mathrm{~d} P \\
W_{\mathrm{r}}=-\int_{P_{\mathrm{m}}}^{P_{\mathrm{r}}} E \mathrm{~d} P
\end{gathered}
$$




$$
\eta=W_{\mathrm{r}} / W_{\mathrm{s}}
$$

where $P_{\mathrm{m}}$ and $P_{\mathrm{r}}$ are maximum and remanent polarizations, respectively. While the voltage across conventional capacitors decreases linearly as they are discharged, the strongly nonlinear-type capacitors that exhibit the polarization jump can retain their voltage. This characteristic can simplify the electronics required to deliver a constant voltage from a capacitor. In addition, antiferroelectrics can store energy at a high density more effectively than linear dielectrics and ferroelectrics. Especially high performance has been achieved with leadcontaining antiferroelectrics, ${ }^{12-14}$ as exemplified by $(\mathrm{Pb}, \mathrm{La})(\mathrm{Zr}, \mathrm{Ti}) \mathrm{O}_{3} \quad$ (PLZT) compounds, which have been commercially used in dc link condensers. Also, extensive research has led to remarkable improvements in the electric storage performance of lead-free alternatives. ${ }^{15-18}$ Ultrahigh energy storage has also been achieved by modifying these antiferroelectrics into relaxors. ${ }^{19-22}$

Organic molecular compounds have additional advantages when used in energy storage devices, such as mechanical flexibility, low density, and environmental benignity, as well as good dielectric strength. For instance, free-standing polycrystalline ferroelectric films have been prepared from small organic molecules. ${ }^{23}$ Over the past decade, we have discovered antiferroelectric switching or analogous metaelectric transitions in several hydrogen-bonded compounds. ${ }^{24-27}$ Highly efficient energy storage in a squaric acid (SQA) crystal, which comprises an antiparallel array of polar sheets, has been highlighted. The molecular dipole moments are reoriented through proton tautomerism (also known as prototropy), which simultaneously relocates the double bonds of a $\pi$-conjugated system and a proton of the adjacent hydrogen bond. ${ }^{26}$ The next challenge is to increase the stored energy density, which requires increasing the polarization jump $\Delta P$ and the switching field $E_{\mathrm{sw}}$.

Here, we develop a series of prototropic organic dielectrics having antipolar or canted electric dipoles. Excellent polarization performance, with a $\Delta P$ exceeding $15 \mu \mathrm{C} \mathrm{cm}^{-2}$, is achieved by improving the dielectric strength of SQA and by preparing new antiferroelectrics: deuterated SQA- $d_{2}$ and furan-3,4dicarboxylic acid (FDC). The materials development is accompanied by the discovery of multiple phase changes in another new antiferroelectric: 3-(4-chlorophenyl)propiolic acid (CPPLA). The alternative approach to antiferroelectric phase switching is to exploit the crystal anisotropy of a ferroelectric having canted dipoles. For each prototropic antiferroelectric investigated, the polarization of the polar subunit (i.e., a hydrogen-bonded sheet or chain) is theoretically simulated and its simple flipping model is examined to explain the polarization jump. Record high energy-storage performances are also clarified in comparison with the corresponding performances of inorganic antiferroelectrics.

\section{Results and discussion}

\section{SQA and deuterated SQA- $d_{2}$}

The SQA crystal ${ }^{28-30}$ is a layered antiferroelectric at temperatures less than $373 \mathrm{~K}$. Its structure belongs to the monoclinic $P 2_{1} / \mathrm{m}$ space group with pseudotetragonal symmetry. The twodimensional hydrogen-bonding network constructs dipolar molecular sheets, the polarities of which alternate along the $c_{\text {tetra-direction. Although previous polarization hysteresis }}$ experiments $^{26}$ showed that the best field-induced polarization was achieved at that time, their maximum field amplitude was set at $\sim 150 \mathrm{kV} \mathrm{cm}{ }^{-1}$ to avoid electric breakdown of the test single crystal. Higher-quality single crystals enabled us to increase the maximum field strength (to $220-230 \mathrm{kV} \mathrm{cm}^{-1}$ ) in this re-examination of the SQA and to conduct new tests on the deuterated SQA- $d_{2}$. In Fig. $1 \mathrm{~b}$ and $\mathrm{c}$, the entire switching process is shown together with a steep polarization jump in the corresponding $P-E$ curve and a sharp peak in the corresponding current density $(J)-E$ curve. With an $\boldsymbol{E} \|[100]_{\text {tetra }}$ configuration at room temperature, the polarization jump $\Delta P$ was optimized to 17.2 and $18.4 \mu \mathrm{C} \mathrm{cm}{ }^{-2}$ for the SQA and SQA- $d_{2}$ crystals, respectively. These polarizations are greatly improved
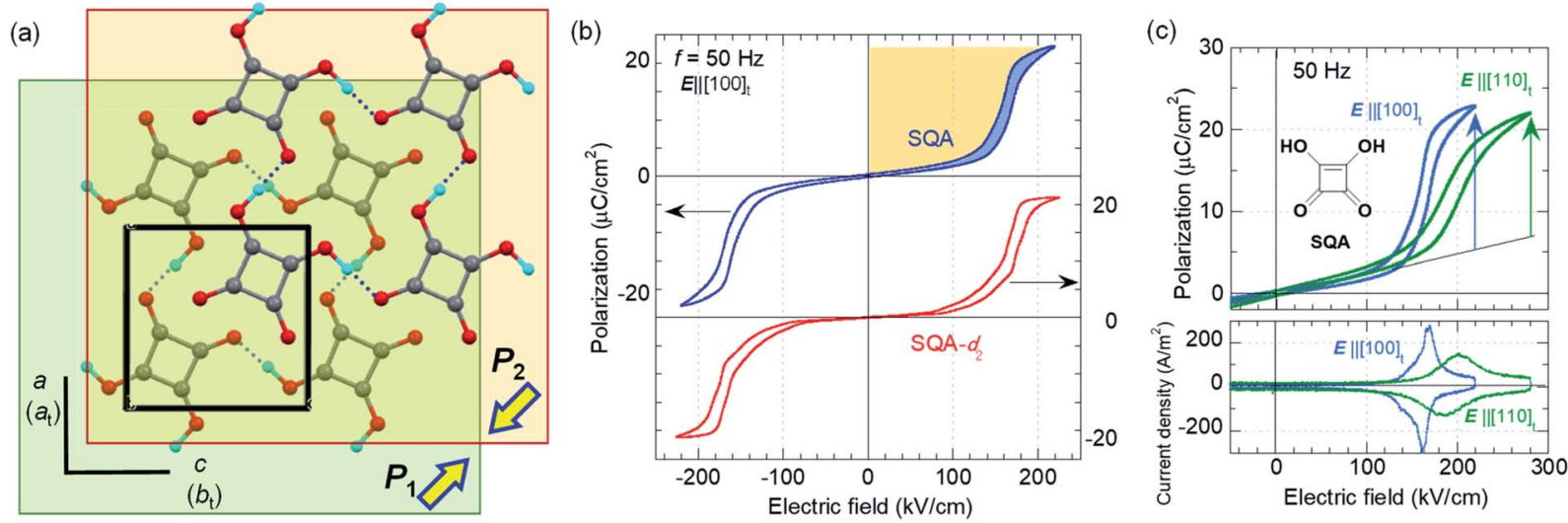

Fig. 1 Squaric acid (SQA): (a) crystal structure viewed along the $b$ ( $c_{\text {tetra }}$ ) direction. Arrows point to the directions of sheet (sublattice) polarizations $P_{1}$ and $P_{2}$. (b) Electric polarization $(P)$ vs. electric field $(E)$ hysteresis loops for SQA and its deuterated SQA- $d_{2}$ crystals at room temperature. Orange and blue areas correspond to the recoverable energy density $W_{\mathrm{r}}$ (orange) and the unrecoverable energy density $W_{\text {loss }}$ (blue area). (c) $P-E$ hysteresis loops and corresponding $J-E$ curves with $E \|\langle 100\rangle_{\text {tetra }}$ and $E \|\langle 110\rangle_{\text {tetra }}$ configurations for an SQA crystal at room temperature and at $f$ $=50 \mathrm{~Hz}$. 
compared with that previously reported for SQA $(10.5 \mu \mathrm{C}$ $\left.\mathrm{cm}^{-2}\right)^{26}$ and are the largest polarizations reported for organic molecular antiferroelectric compounds.

Immediately after the discovery of antiferroelectricity, Kittel introduced the Landau-type macroscopic model comprising two interpenetrating sublattices with opposite polarizations. ${ }^{7}$ For the antiferroelectrics and a dipole-canted ferroelectric examined herein, the sublattices can also be defined by the periodic array of the polar subunits (chains or sheets) of identical polarities. The SQA crystal contains two sublattices with polarizations $\boldsymbol{P}_{1}$ and $\boldsymbol{P}_{2}\left(=-\boldsymbol{P}_{1}\right)$ being parallel to the $\langle\mathbf{1 1 0}\rangle_{\text {tetra }}$ direction. As theoretically simulated elsewhere, ${ }^{31}$ the external field induces a $90^{\circ}$ rotation of $\boldsymbol{P}_{1}$ or $\boldsymbol{P}_{2}$, causing the polarization jump $\Delta P=\sqrt{2}\left|P_{1}\right|\left(16.4 \mu \mathrm{C} \mathrm{cm}^{-2}\right)$ along the $\langle 100\rangle_{\text {tetra }}$ direction, rather than a $180^{\circ}$ flip of $\boldsymbol{P}_{1}$ or $\boldsymbol{P}_{2}$, which would give $\Delta P=2\left|\boldsymbol{P}_{1}\right|$ $\left(23.2 \mu \mathrm{C} \mathrm{cm}^{-2}\right.$ ) along the $\langle 110\rangle_{\text {tetra }}$ direction. Notably, this interpretation does not change under this revision because the easy switching axes are $\langle 100\rangle_{\text {tetra }}$ and the observed polarization jump $\Delta P$ is very similar to $\sqrt{2}\left|P_{1}\right|$, as clearly shown in Fig. 1c.

\section{Furan-3,4-dicarboxylic acid (FDC) with large polarization}

While the polar crystal structures of ferroelectrics usually reveal some additional hidden crystal symmetries (pseudosymmetries), similar key signatures are available for researchers searching for new antiferroelectric candidates. Here, we have discovered the new antiferroelectric furan-3,4-dicarboxylic acid (FDC) crystals, which exhibit the second-best polarization jump among organic antiferroelectrics. In the Cambridge Structure Database, all three available datasets concern the identical monoclinic polymorph (denoted as the $\alpha$-form hereafter) grown from aqueous solution. In the first structural analysis (ref code:
FURDCB), Williams et al. found that both acidic protons were disordered by crystal symmetry (space group $P 2_{1} / m$ ). ${ }^{32}$ Later, Semmingsen et al. redetermined the crystal structure at $T=125$ $\mathrm{K}$ and found that the appearance of weak Bragg spots was caused by the antipolar arrangement of protons with twofold periodicity along the $c$-axis (ref code: FURDCB01). ${ }^{33}$ Our careful reassessment confirmed the validity of the latter structure even at room temperature. The $c / 2$-translation symmetry is hidden in this antipolar structure. In the presence of pseudosymmetry, the antipolar and polar structures can be interconverted by rearranging the protons with minimalized modulation of the host lattice. The actual crystal structure belongs to the $P 2_{1} / c$ space group (\#14). The global crystal symmetry except the two protons has a mirror plane normal to the molecular plane, in addition to the aforementioned $c / 2$-translation. The hydrogenbonded molecular chains parallel to the $b$-direction have longitudinal dipoles whose polarities alternate along the $c$ direction.

Two additional polymorphs were newly generated by sublimation under reduced pressure: rhombus plates of $\beta$-FDC (major form; see ESI Fig. S1 $\uparrow$ for detailed molecular packing with orthorhombic Pbcm symmetry) and rectangular plates of $\gamma$ FDC (minor form; triclinic). Although both crystal forms are similar to $\alpha$-FDC with respect to their hydrogen-bonded molecular sequence (Fig. S2 $\dagger$ ), polarization switching is hardly expected in the $\beta$-FDC crystal, which lacks the corresponding pseudosymmetry.

The crystal structure of $\gamma$-FDC exhibits a $C$-centered monoclinic lattice but exhibits only inversion symmetry. Instead of the unconventional space group $C \overline{1}$, we used the equivalent triclinic space group $P \overline{1}$ for the structural determination. The $a /$ 2-translation symmetry is hidden in this antipolar structure.
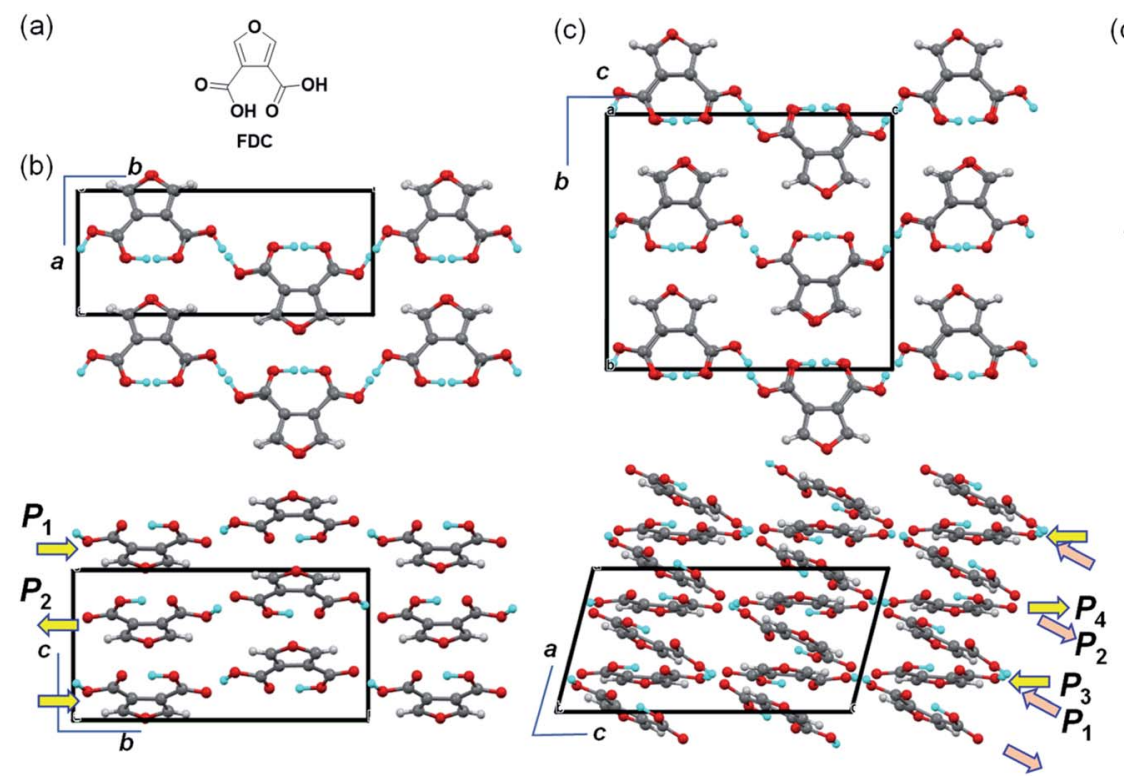

(d)

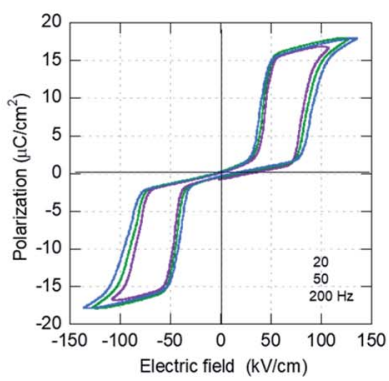

Fig. 2 Furan-3,4-dicarboxylic acid (FDC): (a) chemical structure. (b) Molecular arrangements in the $\alpha$-form crystal viewed along the stacking c(top) and a-directions (bottom). (c) Molecular arrangements in the $\gamma$-form crystal viewed along the stacking $a$ - (top) and $b$-directions (bottom). Arrows represent the polarity of the hydrogen-bonded chains. (d) $P-E$ hysteresis loops with $E \|[\overline{1} 02]$ configurations in the $\gamma$-FDC crystal at room temperature and at various frequencies. 
The large unit cell of the $\gamma$-FDC crystal accommodates four sublattices $\boldsymbol{P}_{1}, \boldsymbol{P}_{2}\left(=-\boldsymbol{P}_{1}\right), \boldsymbol{P}_{3}$, and $\boldsymbol{P}_{4}\left(=-\boldsymbol{P}_{3}\right)$. Therefore, flipping $\boldsymbol{P}_{1}$ and $\boldsymbol{P}_{3}$ (or $\boldsymbol{P}_{2}$ and $\boldsymbol{P}_{4}$ ) gives rise to $\Delta P=\left|2 \boldsymbol{P}_{1}+2 \boldsymbol{P}_{3}\right|$.

We demonstrated the expected phase-change phenomena described in the preceding paragraph for the $\gamma$-FDC crystal (Fig. 2d). The results of $P-E$ hysteresis measurements with the applied ac electric field configuration $E \|[102]$ show the double loop characteristic of the antiferroelectric-ferroelectric transition. The observed polarization jump $\Delta P$, which was as large as $15.1 \mu \mathrm{C} \mathrm{cm}^{-2}$, is slightly smaller than those of SQA but the second-highest among those of organic antiferroelectric crystals. The switching at $\sim 70 \mathrm{kV} \mathrm{cm}^{-1}$ is accompanied by a large hysteresis width of $\sim 50 \mathrm{kV} \mathrm{cm}^{-1}$.

Although the $\alpha$-form crystal is predicted to show a similarly large $\Delta P$, its small size precluded satisfactory measurements. For the $\beta$-FDC crystal, no signatures of phase-change phenomena were detected at least up to $180 \mathrm{kV} \mathrm{cm}^{-1}$ in the measurements at room temperature and at $80{ }^{\circ} \mathrm{C}$, as suggested by the aforementioned structural assessments.

\section{Antiferroelectric with multiple switching processes}

We discovered the new antiferroelectric CPPLA crystal while seeking for additional hidden crystal symmetries (pseudosymmetries) in the reported crystal structures. Das et al. reported a polar monoclinic crystal structure for the iodinesubstituted derivative 3-(4-iodophenyl)propiolic acid (ref code: BEFSUB,${ }^{34}$ in which we noticed pseudo-inversion symmetry, suggesting a possible ferroelectric. They also reported paraelectric-like crystal structures of CPPLA, in which the hydrogen-bonded molecular sequence pinpoints the disordered (or centered) hydrogen atom on each hydrogen bond because of inversion symmetry (ref code: SUHSET). ${ }^{34,35}$ In contrast, our reexamination of the single-crystal structure by X-ray diffraction revealed additional weak Bragg spots indicative of the doubled periodicity as $(\boldsymbol{a}, \boldsymbol{b}, \boldsymbol{c})=\left(-\boldsymbol{a}_{\mathrm{p}}-\boldsymbol{c}_{\mathrm{p}},-\boldsymbol{a}_{\mathrm{p}}+\boldsymbol{c}_{\mathrm{p}}, \boldsymbol{b}_{\mathrm{p}}\right)$. The unit-cell doubling is caused by the antipolar ordering of asymmetrically located hydrogen atoms in the revised crystal structure, which contains two sublattices with polarizations $\boldsymbol{P}_{1}$ and $\boldsymbol{P}_{2}(=$ $-\boldsymbol{P}_{1}$ ) and thus suggests possible antiferroelectricity along the $b$ direction.

Consistent with this expectation, the $P-E$ curve (top panel of Fig. 3b) shows a double hysteresis loop when the maximum field amplitude of $70 \mathrm{kV} \mathrm{cm}^{-1}$ is applied in the $\boldsymbol{E} \|[110]$ configuration (instead of the ideal $\boldsymbol{E} \| \boldsymbol{b}$ configuration because of the crystal shape). However, the application of a higher field amplitude induced an additional polarization jump, causing a quadruple polarization hysteresis loop. This behavior is the manifestation of field-induced successive phase transitions with the antiparallel dipoles flipped half-by-half. Therefore, the intermediate phase is regarded as the ferrielectric state.

\section{Amphoteric behavior of a dipole-canted ferroelectric}

While most ferroelectric crystals have fully aligned dipoles, the ferroelectric 2-phenylmalondialdehyde (PhMDA) single crystal exhibits a canted arrangement of dipolar chains. ${ }^{36}$ Here, we report amphoteric behavior, where a single or double polarization hysteresis loop is obtained depending on the direction of the external field (Fig. 4a). In the orthorhombic crystal with space group $P n a 2_{1}$, the PhMDA molecules form polar hydrogenbonded chains (Fig. 4b). As indicated by small open arrows in Fig. $4 \mathrm{c}$, each chain has a sublattice polarization $\boldsymbol{P}_{1}$ along the [102] direction or $\boldsymbol{P}_{2}$ along the [102] direction. Here, the crystal symmetry demands $P_{1 x}=-P_{2 x}, P_{1 y}=P_{2 y}=0, P_{1 z}=P_{2 z}$; the net polarization $2\left|P_{1 z}\right|$ then emerges in the $c$-direction. Specifically, (a)
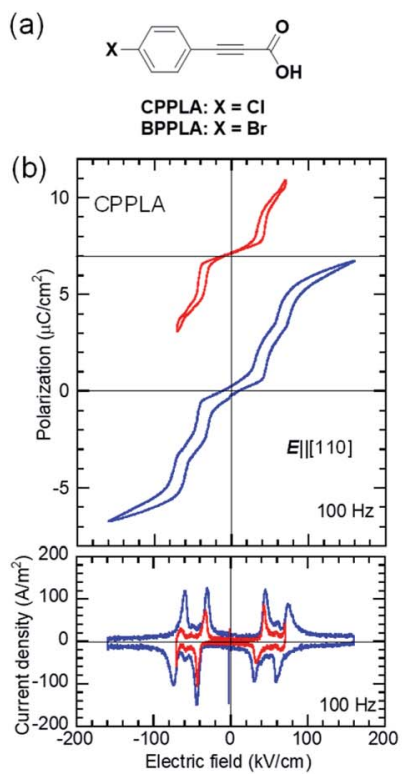

(c)
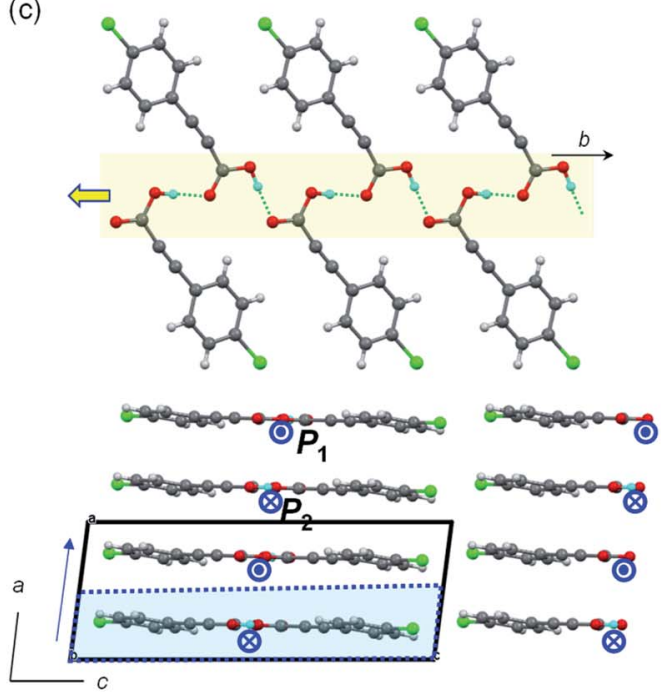

Fig. 3 3-(4-Chlorophenyl)propiolic acid (CPPLA): (a) chemical structure. (b) $P-E$ hysteresis loops obtained with different maximum fields at room temperature (top) and the corresponding $J-E$ curve obtained from the quadruple hysteresis loops, indicating multiple field-induced switching (bottom). (c) A hydrogen-bonded polar molecular ribbon (top) and the crystal structure viewed along the $b$-direction (bottom). Arrows and arrowheads represent the polarity of ribbons. The dotted cell corresponds to the pseudosymmetry of $a / 2$. 

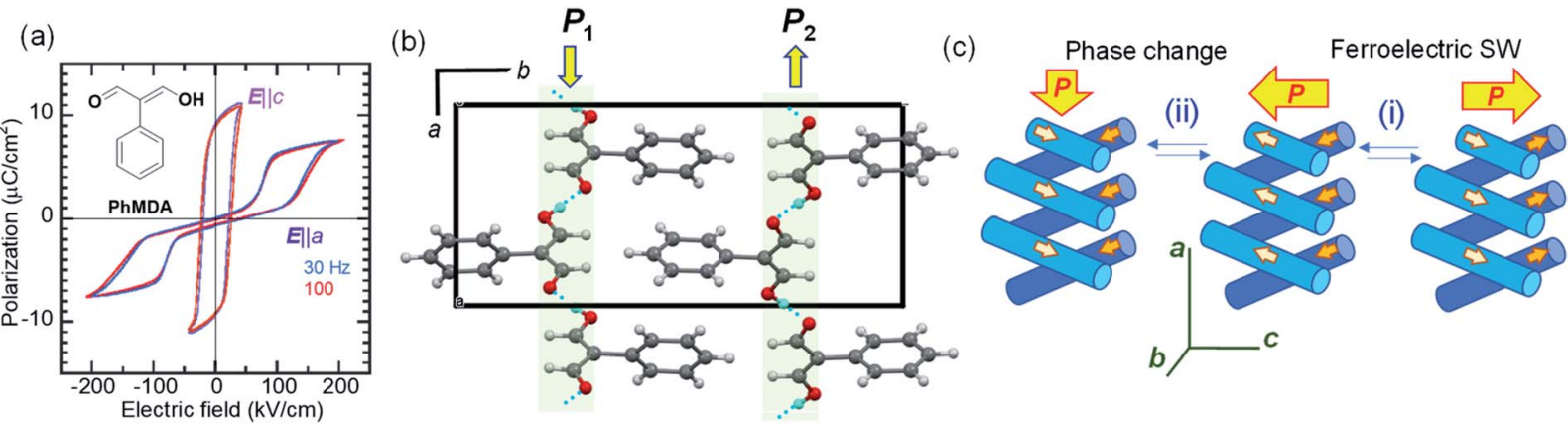

Fig. 4 2-Phenylmalondialdehyde (PhMDA): (a) $P-E$ hysteresis loops at room temperature, as measured with a triangular ac electric field. The applied electric field configurations of $E \| a$ and $E \| c$ are normal and parallel to the spontaneous polarization, respectively. (Inset) The chemical structure of PhMDA. (b) Hydrogen-bonded polar molecular sequences viewed along the c-direction. (c) Schematics of changes in sublattice polarizations (small arrows) of the hydrogen-bonded chains and the total bulk polarization (large arrows) during the (i) ferroelectric polarization reversal and (ii) field-induced phase change.

the configuration of chain dipoles is antiparallel in the $a-$ direction components and parallel in the $c$-direction components. The ferroelectricity observed with the $E \| c$ configuration corresponds to the polarization reversal induced by flipping both $\boldsymbol{P}_{1}$ and $\boldsymbol{P}_{2}$ according to process (i) in Fig. 4c.

In the $\boldsymbol{E} \| \boldsymbol{a}$ configuration, the antiferroelectric-like switching with a $\Delta P$ of $5.8 \mu \mathrm{C} \mathrm{cm}^{-2}$ appears at $\sim 110 \mathrm{kV} \mathrm{cm}^{-1}$, which is substantially greater than the magnitude of the coercive field along the $c$-direction $\left(\sim 20 \mathrm{kV} \mathrm{cm}{ }^{-1}\right)$. The most plausible mechanism is process (ii) in Fig. 4c. The increasing/decreasing electric field flips either $\boldsymbol{P}_{1}$ or $\boldsymbol{P}_{2}$, which causes the observation of $\Delta P_{x}=2\left|P_{1 x}\right|$ through a $90^{\circ}$ rotation of the spontaneous polarization from the the $c$ - to the $a$-direction and vice versa. In this example, the antiferroelectric-like functionalities can be achieved even by rotating polarizations through the fieldinduced transition between two different ferroelectric phases.

\section{Theoretical evaluation of polarization}

The experimentally optimized spontaneous polarizations of organic ferroelectrics have recently been reproduced with excellent accuracy using density functional theory (DFT) calculations and the Berry phase formalism of electric polarization. ${ }^{37}$ Although the corresponding theoretical evaluations are rare for organic antiferroelectrics, SQA represents an example in which the microscopic switching process has been successfully identified in terms of a sublattice polarization model through comparisons with experimental data ${ }^{31}$ In the present work, the sublattice polarizations are simulated for other prototropic antiferroelectrics (FDC, CPPLA, and benzimidazoles). Except for the $\gamma$-FDC crystal, the two sublattices, which interpenetrate each other, construct the antipolar or dipole-canted structure. First, one of them is extracted as a periodic polar crystal lattice and its sublattice polarization $\boldsymbol{P}_{1}$ is computed. Together with its symmetry-related sublattice polarization $\boldsymbol{P}_{2}$, the theoretical polarization $\Delta \boldsymbol{P}^{\text {cal }}$ is calculated by flipping either $\boldsymbol{P}_{1}$ or $\boldsymbol{P}_{2}$. For the $\gamma$-FDC crystal, two of four sublattices were extracted for calculating the crystallographically independent $\boldsymbol{P}_{1}$ and $\boldsymbol{P}_{4}$. The sublattice polarizations were calculated for different degrees of polar distortion $\lambda$ between the reference symmetrized (hypothetical paraelectric, $\lambda=0$ ) and fully polar (ferroelectric, $\lambda$ $=1$ ) configurations. As shown in Fig. S3, $\dagger$ the smooth $\lambda$ dependence confirms the validity of each simulation.

In the $\gamma$-FDC crystal, each hydrogen-bonded chain is highly polarized and the resultant large $\left|\Delta \boldsymbol{P}^{\mathrm{cal}}\right|$ of $13.2 \mu \mathrm{C} \mathrm{\textrm {cm } ^ { - 2 }}$ directed along the [102] is similar to the experimentally observed polarization jump $\left|\Delta \boldsymbol{P}^{\exp }\right|\left(15.1 \mu \mathrm{C} \mathrm{cm}^{-2}\right)$. Note that nearly the same $\left|\Delta \boldsymbol{P}^{\mathrm{cal}}\right|$ values were computed for both the $\alpha$ and $\beta$-forms, which have similar hydrogen-bonded molecular sequences (Fig. S2 $\dagger$ ). Likewise, excellent agreement between $\left|\Delta \boldsymbol{P}^{\mathrm{cal}}\right|$ and $\left|\Delta \boldsymbol{P}^{\exp }\right|$ is confirmed for a series of prototropic antiferroelectrics, as demonstrated in Fig. 5. Regarding the CPPLA crystal, the entire polarization jump of the quadruple hysteresis loops $\left(4.3 \mu \mathrm{C} \mathrm{cm}^{-2}\right)$ is explained well by the full alignment of chain polarizations (the $\boldsymbol{E} \|[110]$-direction component of $\Delta P^{\mathrm{cal}}$ is $4.8 \mu \mathrm{C} \mathrm{cm}^{-2}$ ).

DFT calculations were also carried out for the dipole-canted ferroelectric PhMDA to evaluate its amphoteric behavior quantitatively. The calculated sublattice $\boldsymbol{P}_{1}$ is $(3.0,0.0,4.5) \mu \mathrm{C} \mathrm{cm}^{-2}$, yielding its counterpart $P_{2}=(-3.0,0.0,4.5) \mu \mathrm{C} \mathrm{cm}{ }^{-2}$ by symmetry. The resultant spontaneous polarization $\boldsymbol{P}_{\mathrm{s}}=\boldsymbol{P}_{1}+\boldsymbol{P}_{2}=$ $\left(0,0,2\left|P_{1 z}\right|\right)$ is $(0,0,9.0) \mu \mathrm{C} \mathrm{cm}^{-2}$ and coincides with the

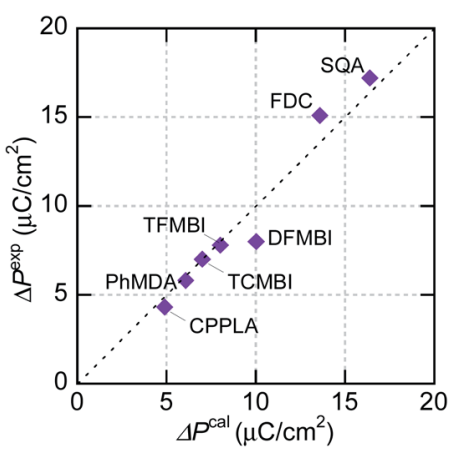

Fig. 5 Comparison of field-induced polarizations of prototropic antiferroelectrics and an amphoteric dielectric, PhMDA. Experimentally observed polarization jump $\left|\Delta P^{\exp }\right|$ at room temperature versus calculated total polarization $\left|\Delta P^{\text {cal }}\right|$ assuming a fully aligned sublattice polarization. 
Table 1 Polarization switching and energy-storage properties of prototropic organic molecular crystals at room temperature

\begin{tabular}{|c|c|c|c|c|c|c|c|c|}
\hline \multirow[b]{2}{*}{ Compound $^{a}$} & Dipole moment of the sublattice & \multirow{2}{*}{ 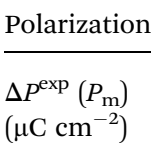 } & \multirow{2}{*}{$\begin{array}{l}\text { Switching field } \\
E_{\mathrm{sw}}\left(\Delta E_{\mathrm{sw}}\right)\left(\mathrm{kV} \mathrm{cm}^{-1}\right)\end{array}$} & \multirow{2}{*}{$\begin{array}{l}\text { Stored energy } \\
W_{\mathrm{r}}\left(W_{\mathrm{s}}\right)\left(\mathrm{J} \mathrm{cm}^{-3}\right)\end{array}$} & \multirow{2}{*}{$\begin{array}{l}\text { Efficiency } \\
\eta\end{array}$} & \multicolumn{3}{|c|}{ Hysteresis conditions ${ }^{b}$} \\
\hline & $\boldsymbol{\mu}(\mathrm{D} \text { per molecule })^{c}$ & & & & & $f(\mathrm{~Hz})$ & $\begin{array}{l}E_{\mathrm{m}} \\
\left(\mathrm{kV} \mathrm{cm}^{-1}\right)\end{array}$ & $\boldsymbol{E}$-direction \\
\hline SQA & $(4.37,0.00,4.93)$ & $17.2(22.7)$ & $166(6)$ & $3.29(3.67)$ & 0.90 & 50 & 210 & $\|\langle 100\rangle_{\text {tetra }}$ \\
\hline $\mathrm{SQA}^{d}$ & & $10.5(13.3)$ & $124(4.6)$ & $1.44(1.53)$ & 0.94 & 100 & 151 & $\|\langle 100\rangle_{\text {tetra }}$ \\
\hline SQA- $d_{2}$ & & $18.4(21.1)$ & $178(20)$ & $3.13(3.49)$ & 0.90 & 50 & 225 & $\|\langle 100\rangle_{\text {tetra }}$ \\
\hline $\mathrm{DFMBI}^{e}$ & $(0.04,0.00,-5.72)$ & $8.0(10.5)$ & $67(12)$ & $0.52(0.67)$ & 0.78 & 2 & 86 & $\|[001]$ \\
\hline TFMBI $^{e}$ & $(0.36,-0.01,-5.04)$ & $7.8(9.0)$ & $13(8)$ & $0.060(0.137)$ & 0.44 & 0.2 & 23 & $\|[001]$ \\
\hline TCMBI $^{e}$ & $(-5.31,0.04,0.00)$ & $7.0(9.4)$ & $49(22)$ & $0.33(0.54)$ & 0.62 & 10 & 80 & $\|[100]$ \\
\hline CPPLA & $(1.43,3.11,0.29)$ & $4.3(6.7)$ & 55 & $0.38(0.50)$ & 0.76 & 100 & 160 & $\|[110]$ \\
\hline PhMDA & $(3.33,0.00,4.95)$ & $5.8(7.5)$ & $110(59)$ & $0.59(1.00)$ & 0.59 & 100 & 200 & $\|[100]$ \\
\hline
\end{tabular}

${ }^{a}$ TFMBI $=2$-trifluoromethylbenzimidazole, DFMBI $=2$-difluoromethylbenzimidazole, TCMBI $=2$-trichloromethylbenzimidazole. ${ }^{b} f=$ applied frequency of triangular waves, $E_{\mathrm{m}}=$ maximum field amplitude applied. ${ }^{c}$ Dipole moment $\mu_{\mathrm{i}}$ was calculated from theoretical sublattice polarization $P_{\mathrm{i}}{ }^{d}$ The data from ref. 26 for SQA. ${ }^{e}$ The data in ref. 24 for three benzimidazoles was used in the analysis.

experimental $P_{\mathrm{S}}\left(9 \mu \mathrm{C} \mathrm{cm}^{-2}\right)$ emerging in the $c$-direction. With the $\boldsymbol{E} \| \boldsymbol{a}$ configuration, the predicted polarization jump $\Delta P_{x}=2\left|P_{1 x}\right|=$ $6.1 \mu \mathrm{C} \mathrm{cm}^{-2}$ agrees well with the experimental $\Delta P$ of $5.8 \mu \mathrm{C} \mathrm{cm}^{-2}$.

\section{Energy-storage performance}

The $P-E$ curves of FDC, CPPLA, and PhMDA crystals were also measured at higher temperatures (Fig. S4 $\dagger$ ). The FDC crystal revealed very weak temperature dependence up to $420 \mathrm{~K}$. For CPPLA and PhMDA as well as for SQA, ${ }^{26}$ both the $\Delta P$ and switching field decrease with heating, and thus the stored energy densities are diminished from the room-temperature performance. Regarding the energy-storage performance of all of the prototropic antiferroelectrics, Table 1 summarizes the room-temperature performance obtained according to eqn (1)(3). Fig. 6 shows a plot of these data in comparison with those of
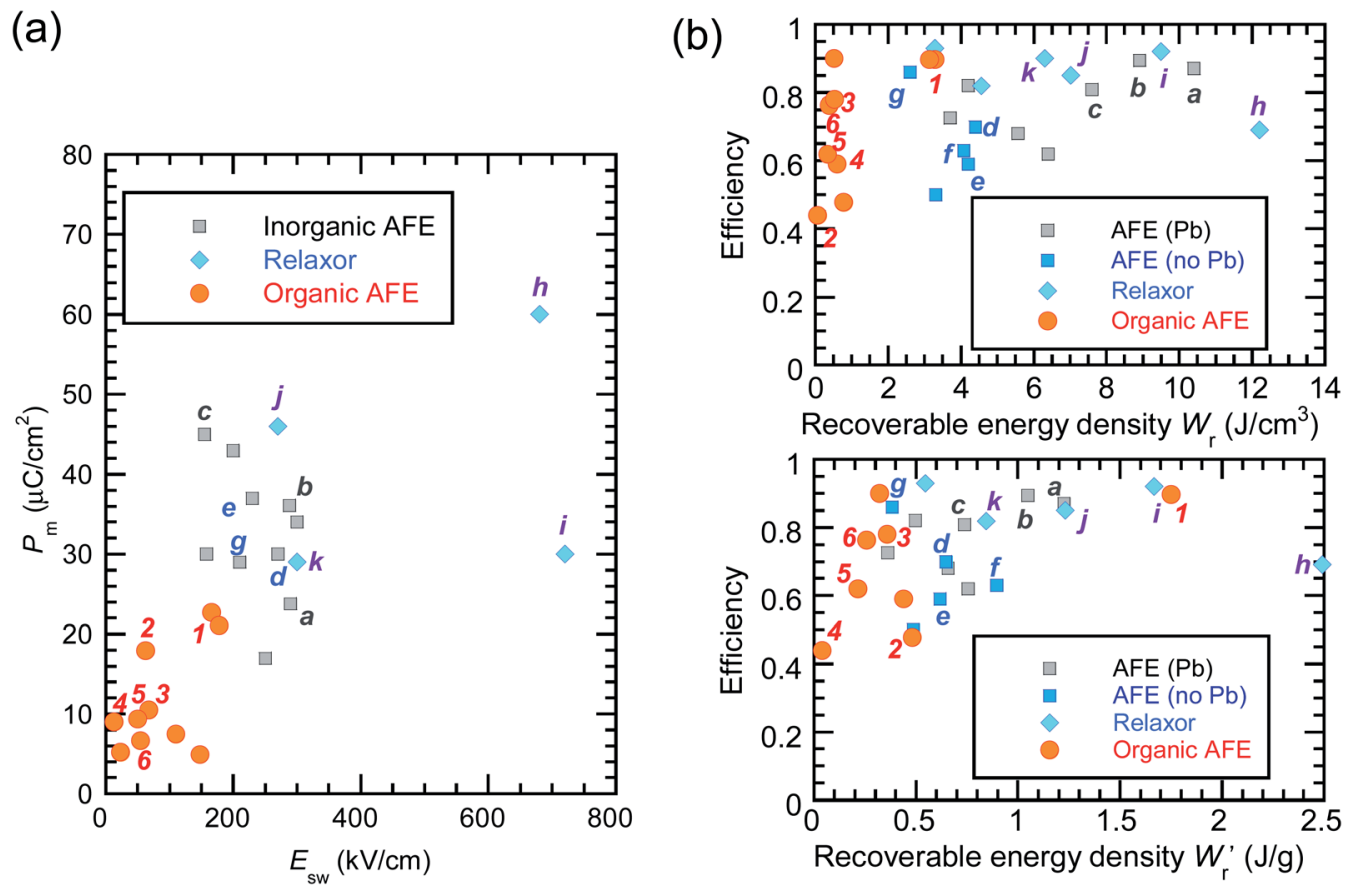

Fig. 6 Energy-storage performance of organic molecular antiferroelectric (AFE) crystals at room temperature in comparison with reported performance for high-performing inorganic AFE or relaxor ceramics. (a) The field-induced maximum polarization vs. switching field. (b) The energy storage efficiency versus the recoverable energy density per unit volume (top) or weight (bottom). Bold alphanumeric characters represent organic antiferroelectrics (1, SQA; 2, $\gamma$-FDC; 3: DFMBI; 4, TFMBI; 5, TCMBI; 6, CPPLA), inorganic antiferroelectrics (a, (Pbo.98-

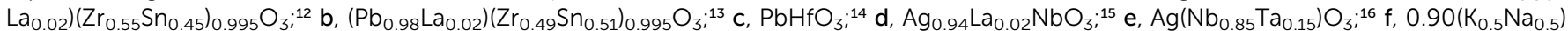

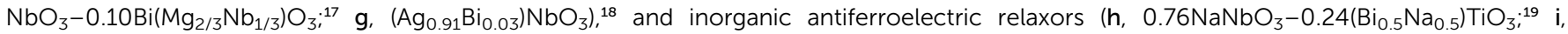
$\left.0.55\left(\mathrm{Bi}_{0.5} \mathrm{Na}_{0.5}\right) \mathrm{TiO}_{3}-0.45\left(\mathrm{Sr}_{0.5} \mathrm{Bi}_{0.5}\right) \mathrm{TiO}_{3},{ }^{20} \mathrm{j}, 0.78\left(\mathrm{Bi}_{0.5} \mathrm{Na}_{0.5}\right) \mathrm{TiO}_{3}-0.22 \mathrm{NaNbO}_{3},{ }^{21} \mathrm{k}, \mathrm{Ag}\left(\mathrm{Nb}_{0.45} \mathrm{Ta}_{0.55}\right) \mathrm{O}_{3}\right) .{ }^{22}$ 
bulk antiferroelectric ceramics and their relaxor modifications, which exhibit high or ultrahigh energy-storage densities. Among the prototropic antiferroelectrics, SQA (and SQA- $d_{2}$ ) crystals exhibit both the highest recoverable energy-storage density $\left(W_{\mathrm{r}}=3.3 \mathrm{~J} \mathrm{~cm}^{-3}\right)$ and nearly ideal efficiency $(\eta=$ 0.90). As shown in Fig. 6a, the excellent $W_{\mathrm{r}}$ of SQA is justified by its best performance with respect to both the maximum polarization $P_{\mathrm{m}}$ and the switching field $E_{\mathrm{sw}}$ (the average of forward and backward phase switching fields). However, the $W_{\mathrm{r}}, P_{\mathrm{m}}$, and $E_{\mathrm{Sw}}$ values are smaller than those of the bulk antiferroelectric ceramics and their relaxor modifications, ${ }^{\mathbf{1 2 - 2 2 , 3 8 - 4 4}}$ as evident in Fig. 6a and b. The magnitude relation of the performance is drastically different in Fig. 6c, which is a replot of the data against the stored energy density per weight $\left(W_{r}^{\prime}\right)$ instead of that per unit volume $\left(W_{\mathrm{r}}\right)$. This difference is attributed to the crystal density $\rho$ of the organic antiferroelectrics (1.3$1.9 \mathrm{~g} \mathrm{~cm}^{-3}$ ) being substantially lower than those $\mathrm{e}^{45}$ of leadcontaining (8.5-10.3 $\left.\mathrm{g} \mathrm{cm}^{-3}\right)$ and lead-free antiferroelectrics $\left(4.6-8.1 \mathrm{~g} \mathrm{~cm}^{-3}\right)$. The $W_{\mathrm{r}}^{\prime}\left(1.75 \mathrm{~J} \mathrm{~g}^{-1}\right)$ of SQA $\left(\rho=1.88 \mathrm{~g} \mathrm{~cm}^{-3}\right)$ exceeds those derived from the highest $W_{\mathrm{r}}$ values (approximately 8-11 $\mathrm{J} \mathrm{cm}^{-3}$ ) reported for several bulk antiferroelectric ceramics $\left(W_{\mathrm{r}}^{\prime}<1.3 \mathrm{~J} \mathrm{~g}^{-1}\right)$. Notably, the SQA crystal retains the highest $\eta$ without resorting to its modification to relaxor forms. These findings are encouraging for the future applications of these materials in portable and/or mobile devices.

\section{Conclusions}

Various dielectric phase-change phenomena have been demonstrated via studies on revisited SQA, newly developed antiferroelectrics, and the "amphoteric dielectric" PhMDA. In terms of the magnitude of the field-induced polarization jump, the best $\left(\sim 18 \mu \mathrm{C} \mathrm{cm}^{-2}\right)$ and second-best performances $(\sim 15 \mu \mathrm{C}$ $\mathrm{cm}^{-2}$ ) among organic antiferroelectrics were achieved. In particular, the improvement of the dielectric strength of SQA resulted in excellent energy-storage performance, including a high recoverable energy-storage density $\left(W_{\mathrm{r}}=3.3 \mathrm{~J} \mathrm{~cm}^{-3}\right)$, while maintaining nearly ideal efficiency $(\eta=90 \%)$. The advantage of organic molecular systems is their low crystal density, which resulted in corresponding energy densities per mass as high as $W_{\mathrm{r}}^{\prime}=1.75 \mathrm{~J} \mathrm{~g}^{-1}$, which exceeds those derived from the highest $W_{\mathrm{r}}$ values $\left(\sim 8-11 \mathrm{~J} \mathrm{~cm}^{-3}\right)$ reported for several bulk antiferroelectric ceramics $\left(W_{\mathrm{r}}^{\prime}<1.3 \mathrm{~J} \mathrm{~g}^{-1}\right)$. Notably, the present SQA crystal has not yet been modified into relaxor forms.

In addition to the antiferroelectrics exhibiting conventional double hysteresis loops, the CPPLA crystal was found to exhibit quadruple polarization hysteresis loops caused by the two-step phase transition with moderate polarization jumps. The ferroelectric PhMDA single crystal represents a novel "amphoteric dielectric" that can exhibit a single or double polarization hysteresis loop depending on the direction of the applied external field relative to the directions of its canted dipole moments. Irrespective of the details of their variation, all of the observed polarizations are consistently explained by the DFT calculations combined with the simplest sublattice model. Such a theoretically precise prediction ability provides a powerful tool for improving the energy-storage performance; it will accelerate the materials design and exploration as well as deepening our understanding of the microscopic phase-change mechanisms.

\section{Experimental section}

\section{Preparation and electric measurements}

Commercially available SQA, FDC, PhMDA, and CPPLA were purified by repeated recrystallizations and/or temperaturegradient vacuum sublimation. The temperature-gradient sublimation under reduced pressure gave single crystals with shapes of elongated rectangular plates in the case of FDC, parallelogram-shaped plates in the case of CPPLA, and thick plates in the case of PhMDA. The bipyramidal crystals of SQA and SQA- $d_{2}$, which were grown by recrystallization from hot deionized water and $99.5 \% \mathrm{D}_{2} \mathrm{O}$, respectively, were cut with a blade for electric measurements according to previously reported procedures. ${ }^{26}$ All of the electric measurements were conducted using single crystals with painted silver electrodes. The $P-E$ hysteresis measurements were performed by applying a high-voltage triangular wave field and various alternating frequencies to single crystals, which were immersed in silicone oil to prevent atmospheric discharge. The system used to evaluate ferroelectrics (Toyo Corporation, FCE-1) comprised a current/charge-voltage converter (model 6252), an arbitrary waveform generator (Biomation 2414B), an analog-to-digital converter (WaveBook 516), and a voltage amplifier (NF Corporation, HVA4321).

\section{Crystallographic studies}

The crystallographic data and experimental details are summarized in Table S1.†X-ray diffraction data were collected from single crystals at room temperature using graphitemonochromated Mo $\mathrm{K} \alpha$ radiation $(\lambda=0.7107 \AA)$ and a fourcircle diffractometer equipped with a two-dimensional detector [hybrid pixel detector (Rigaku AFC10 with PILATUS200K)]. CrystalStructure crystallographic software packages [Molecular Structure Corp. (MSC; Woodlands, TX) and Rigaku Corp. (Tokyo)] were used for the direct method and for the refinement of the structures. Final refinements of the nonhydrogen atoms were performed with anisotropic thermal factors. The hydrogen-bonded hydrogen atoms were found by differential Fourier synthesis and were refined isotropically; the remaining hydrogen atoms were calculated in their ideal geometrical positions.

\section{Theoretical calculations}

First, for the experimentally obtained AFE structures, hydrogen positions were computationally optimized to minimize the total energy. Next, for each system, all of the atoms except for one polar subunit were removed from the unit cell $(\lambda=1)$. Reference nonpolar structures $(\lambda=0)$ were constructed by symmetrization. The polarization as a function of $\lambda$ was calculated using the Berry phase approach. ${ }^{46,47}$ All the calculations were performed using the QMAS code ${ }^{48}$ based on the projector augmented-wave $\operatorname{method}^{49}$ and the plane-wave basis set. To describe the 
electronic exchange-correlation energy, the Perdew-BurkeErnzerhof (PBE) version of the generalized gradient approximation (GGA) ${ }^{50}$ was used.

\section{Author contributions}

S. H. prepared the purified single crystals, performed the dielectric measurements, conceived the study design, and wrote most of the paper. S. I performed the theoretical calculations.

\section{Conflicts of interest}

There are no conflicts to declare.

\section{Acknowledgements}

This work was partially supported by JSPS KAKENHI Grant Number 21H04679 and JST CREST Grant Number JPMJCR18J2, Japan. Part of the computation in this work was done using the facilities of the Supercomputer Center, the Institute for Solid State Physics, the University of Tokyo.

\section{Notes and references}

1 M. E. Lines and A. M. Glass, Principles and Applications of Ferroelectrics and Related Materials, Oxford University Press, New York, 1977.

2 K. Uchino and J. R. Giniewicz, Micromechatronics, Marcel Dekker, New York, 2003.

3 Z. Yao, Z. Song, H. Hao, Z. Yu, M. Cao, S. Zhang, M. T. Lanagan and H. Liu, Adv. Mater., 2017, 29, 1601727.

4 H. Palneedi, M. Peddigari, G.-T. Hwang, D.-Y. Jeong and J. Ryu, Adv. Funct. Mater., 2018, 28, 1803665.

5 Z. Liu, T. Lu, J. Ye, G. Wang, X. Dong, R. Withers and Y. Liu, Adv. Mater. Tech., 2018, 3, 1800111.

6 L. Yang, X. Kong, F. Li, H. Hao, Z. Cheng, H. Liu, J.-F. Li and S. Zhang, Prog. Mater. Sci., 2019, 102, 72-108.

7 C. Kittel, Phys. Rev., 1951, 82, 729-732.

8 E. Sawaguchi, H. Maniwa and S. Hoshino, Phys. Rev., 1951, 83, 1078.

9 G. Shirane, E. Sawaguchi and Y. Takagi, Phys. Rev., 1951, 84, 476-481.

10 X. Tan, C. Ma, J. Frederick, S. Beckman and K. G. Webber, J. Am. Ceram. Soc., 2011, 94, 4091-4107.

11 X. Hao, J. Zhai, L. B. Kong and Z. Xu, Prog. Mater. Sci., 2014, 63, 1-57.

12 H. Wang, Y. Liu, T. Yang and S. Zhang, Adv. Funct. Mater., 2019, 29, 1807321.

13 Y. Liu, S. Liu, T. Yang and H. Wang, J. Mater. Sci., 2021, 56, 6073-6082.

14 J. Wei, T. Yang and H. Wang, J. Eur. Ceram. Soc., 2019, 39, 624-630.

15 J. Gao, Y. C. Zhang, L. Zhao, K. Y. Lee, Q. Liu, A. Studer, M. Hinterstein, S. J. Zhang and J. F. Li, J. Mater. Chem. A, 2019, 7, 2225-2232.

16 L. Zhao, Q. Liu, J. Gao, S. Zhang and J. F. Li, Adv. Mater., 2017, 29, 1701824.
17 T. Q. Shao, H. L. Du, H. Ma, S. B. Qu, J. Wang, J. F. Wang, W. Y. Wei and Z. Xu, J. Mater. Chem. A, 2017, 5, 554-563.

18 Y. Tian, L. Jin, H. F. Zhang, Z. Xu, X. Y. Wei and G. Viola, J. Mater. Chem. A, 2017, 5, 17525-17531.

19 H. Qi, R. Zuo, A. Xie, A. Tian, J. Fu, Y. Zhang and S. Zhang, Adv. Funct. Mater., 2019, 29, 1903877.

$20 \mathrm{~J} . \mathrm{Li}, \mathrm{F} . \mathrm{Li}, \mathrm{Z} . \mathrm{Xu}$ and X. Zhang, Adv. Mater., 2018, 30, 1802155.

21 K. Zou, Y. Dan, H. Xu, Q. Zhang, Y. Lu, H. Huang and Y. He, Mater. Res. Bull., 2019, 113, 190-210.

22 N. Luo, K. Han, M. J. Cabral, X. Liao, S. Zhang, C. Liao, G. Zhang, X. Chen, Q. Feng, J. F. Li and Y. Wei, Nat. Commun., 2020, 11, 4824.

23 J. Harada, APL Mater., 2021, 9, 020901.

24 S. Horiuchi, F. Kagawa, K. Hatahara, K. Kobayashi, R. Kumai, Y. Murakami and Y. Tokura, Nat. Commun., 2012, 3, 1308.

25 K. Kobayashi, S. Horiuchi, S. Ishibashi, R. Murakami and R. Kumai, J. Am. Chem. Soc., 2018, 140, 3842-3845.

26 S. Horiuchi, R. Kumai and S. Ishibashi, Chem. Sci., 2018, 9, 425-432.

27 S. Horiuchi, S. Ishibashi, R. Haruki, R. Kumai, S. Inada and S. Aoyagi, Chem. Sci., 2020, 11, 6183-6192.

28 D. Semmingsen, Acta Chem. Scand., 1973, 27, 3961-3972.

29 J. Feder, in, Oxocarbons, ed. R. West, Academic Press, New York, 1980, pp. 141-167.

30 Y. Moritomo, Y. Tokura, H. Takahashi and Y. Mori, Phys. Rev. Lett., 1991, 67, 2041-2044.

31 S. Ishibashi, S. Horiuchi and R. Kumai, Phys. Rev. B, 2018, 97, 184102.

32 D. E. Williams and R. E. Rundle, J. Am. Chem. Soc., 1964, 86, 1660-1666.

33 D. Semmingsen, S. Nordenson and A. Aasen, Acta Chem. Scand., Ser. A, 1986, 40, 559-565.

34 D. Das, R. K. R. Jetti, R. Boese and G. R. Desiraju, Cryst. Growth Des., 2003, 3, 675-681.

35 G. R. Desiraju, B. N. Murty and K. V. R. Kishan, Chem. Mater., 1990, 2, 447-449.

36 S. Horiuchi, R. Kumai and Y. Tokura, Adv. Mater., 2011, 23, 2098-2103.

37 S. Horiuchi, K. Kobayashi, R. Kumai and S. Ishibashi, Nat. Commun., 2017, 8, 14426.

38 Q. Zhang, H. Tong, J. Chen, Y. Lu, T. Yang, X. Yao and Y. He, Appl. Phys. Lett., 2016, 109, 262901.

39 J. Shen, X. Wang, T. Yang, H. Wang and J. Wei, J. Alloys Compd., 2017, 721, 191-198.

40 L. Zhang, S. Jiang, B. Fan and G. Zhang, J. Alloys Compd., 2015, 622, 162-165.

41 P. Gao, Z. Liu, N. Zhang, H. Wu, A. A. Bokov, W. Ren and Z.-G. Ye, Chem. Mater., 2019, 31, 979-990.

42 L. Zhao, J. Gao, Q. Liu, S. Zhang and J. F. Li, ACS Appl. Mater. Interfaces, 2018, 10, 819-826.

43 Y. Tian, L. Jin, H. Zhang, Z. Xu, X. Wei, E. Politova, S. Y. Stefanovich, N. V. Tarakina, I. Abrahams and H. Yan, J. Mater. Chem. A, 2016, 4, 17279-17287. 
44 Q. Li, C. Zhou, J. Xu, L. Yang, X. Zhang, W. Zeng, C. Yuan, G. Chen and G. Rao, J. Mater. Sci.: Mater. Electron., 2016, 27, 10810-10815.

45 Landolt-Börnstein Numerical Data and Functional Relationships in Science and Technology, New Series, Group III: Crystal and Solid State Physics, Ferroelectric and Related Substances, ed. Y. Shiozaki, E. Nakamura and T. Mitsui, Springer-Verlag, Berlin, 2006, vol. 36.

46 R. Resta, Rev. Mod. Phys., 1994, 66, 899-915.
47 R. D. King-Smith and D. Vanderbilt, Phys. Rev. B: Condens. Matter Mater. Phys., 1993, 47, 1651-1654.

48 S. Ishibashi, T. Tamura, S. Tanaka, M. Kohyama and K. Terakura, Phys. Rev. B: Condens. Matter Mater. Phys., 2007, 76, 153310.

49 P. E. Blöchl, Phys. Rev. B: Condens. Matter Mater. Phys., 1994, 50, 17953-17979.

50 J. P. Perdew, K. Burke and M. Ernzerhof, Phys. Rev. Lett., 1996, 77, 3865-3868. 